\title{
Iris Recognition with Support Vector Machines
}

\author{
Kaushik Roy and Prabir Bhattacharya \\ Concordia Institute for Information System Engineering, \\ Concordia University, Montreal, Quebec, Canada H3G 1M8 \\ \{kaush_ro, prabir\}@ciise.concordia.ca
}

\begin{abstract}
We propose an iris recognition system for the identification of persons using support vector machines. Canny's edge detection and the Hough transform are used to find the iris/pupil boundary and a simple thresholding method is employed for eyelash detection. The Gabor wavelet technique is deployed in order to extract the deterministic features in the transformed iris of a person in the form of template. The extracted iris features are fed into a support vector machine (SVM) for classification. Our results indicate that the performance of SVM as a classifier is far better than the performance of a classifier based on the artificial neural network.
\end{abstract}

\section{Introduction}

To meet the increasing security requirements of the present-day society, personal identification is becoming more and more important. There has been a lot of interest recently for using the iris recognition technology as a tool in the fight against terrorism and other crimes [1][2][3]. The main concept behind this technique is to use the iris (the colored portion of the eye, around the pupil) as a means of uniquely identifying each person. Each iris is unique and does not change over the person's lifetime, therefore making their usage to identify people works even better than fingerprinting and human eye retina. Iris is a physiological biometric feature and it contains a unique texture which is complex enough to be used as a biometric signature. In this work, an iris recognition system is developed using supper vector machine (SVM) as a pattern classifier. A novel feature of this paper is the application of SVM for iris classification. Although SVM is widely used in pattern recognition applications [10], this paper is one of the few works on the application of SVM to iris classification except for [8][9].

\section{Pre-processing}

\subsection{Iris Image Segmentation}

The iris region isolation from the digital image of the eye is the first step of iris recognition. Lower and upper parts of the iris region are occluded by the eyelids and eyelashes. An automatic segmentation algorithm based on the circular Hough 


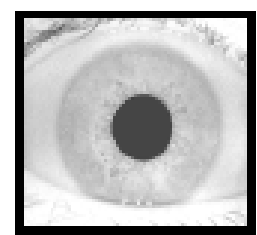

(a)

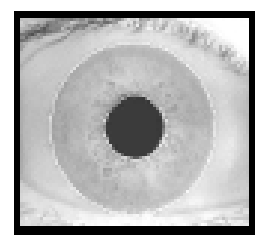

(b)

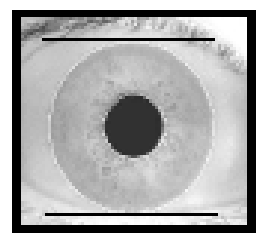

(c)

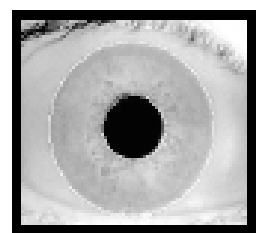

(d)

Fig. 1. (a) Sample iris image (b) Iris and pupil boundary are detected from the sample iris image (c) Top and bottom eyelid detection (d) Eyelash has been detected using a thresholding method and denoted as black

transform is employed here. Firstly, an edge map is generated by calculating the first derivatives of intensity values in an image using canny edge detection and then thresholding the result. From the edge map, votes are cast in the Hough space for the parameters of circles passing through each edge point. A maximum point in the Hough space corresponds to the radius and centre coordinates of the circle best defined by the edge points. To perform the edge detection, the derivatives are biased in horizontal direction to detect the eyelids and outer circular boundary of iris in vertical direction since the eyelids are usually horizontally aligned, and also the eyelid edge map corrupts the circular iris boundary edge map, if using all gradient data. The range of radius values is set manually and the values of the iris radius range from 85 to 140 pixels, while the pupil radius ranges from 25 to 74 pixels. In order to make the circle detection process more efficient and accurate, the Hough transform for the iris/sclera boundary is performed first, then the Hough transform for the iris/pupil boundary is performed within the iris region, instead of the whole eye region, since the pupil is always within the iris region. Fig.1 (a) shows a sample iris image and iris/pupil regions are isolated with circular boundary from this image as shown in Fig.1 (b). Here eyelids are isolated by first fitting a line to the upper and lower eyelid using the linear Hough transform. A second horizontal line is then drawn, which intersects with the first line at the iris edge that is closest to the pupil. This process is illustrated in Fig. 1(c) and is done for both the top and bottom eyelids. The Canny edge detection is used to create an edge map, and only horizontal gradient information is taken. If the maximum in Hough space is lower than a threshold value then no line is fitted since this belongs to non-occluding eyelids. According to Kong and Zhang [6][7], separable eyelashes are detected using 1D Gabor filters, since a low output value is produced by convolution of a separable eyelash with the Gaussian smoothing function. Thus, if a resultant point is smaller than a threshold, it is noted that this point belongs to an eyelash. Multiple eyelashes are detected using the variance of intensity and if the values in a small window are lower than a threshold, the centre of the window is considered as a point in an eyelash as shown in Fig. 1(d).

\subsection{Image Transformation}

Once the iris region is successfully segmented, the next step is to transform the extracted iris region into a fixed dimension for further comparison. The transformation process will produce iris regions, which have the same constant 


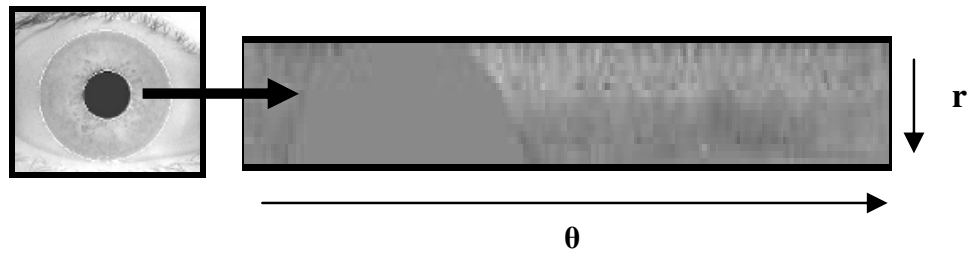

Fig. 2. Transformed Iris Image

dimensions, so that two images of the same iris under different conditions will have characteristic features at the same spatial location. The homogenous rubber sheet model devised by Daugman [2] is used here which remaps each point within the iris region to a pair of polar coordinates. The rubber sheet model takes into account pupil dilation and size inconsistencies to produce a normalized representation with constant dimension. In this way the iris region is modeled as a flexible rubber sheet anchored at the iris boundary with the pupil centre as the reference point. A constant number of points are chosen along each radial line, so that a constant number of radial data points are taken, irrespective of how narrow or wide the radius is at a particular angle. The transformed pattern is created by backtracking to find the Cartesian coordinates of data points from the radial and angular position in the normalized pattern. From the 'doughnut' iris region, it produces a 2D array with horizontal dimensions of angular resolution and vertical dimensions of radial resolution. Another $2 \mathrm{D}$ array is created for marking reflections, eyelashes, and eyelids detected in the segmentation stage. In order to prevent non-iris region data from corrupting the normalized representation, data points which occur along the pupil border or the iris border are discarded. Fig. 2 shows the transformed iris image.

\subsection{Feature Extraction and Encoding}

Feature encoding is implemented by convolving the normalized iris pattern with 1D Log-Gabor wavelets [4]. The 2D normalized pattern is broken up into a number of 1D signals, and then these 1D signals are convolved with 1D Gabor wavelets. The rows of the $2 \mathrm{D}$ normalized pattern are taken as the $1 \mathrm{D}$ signal, each row corresponds to a circular ring on the iris region. The angular direction is taken rather than the radial one, which corresponds to columns of the normalized pattern, since maximum independence occurs in the angular direction. The intensity values at known noise areas in the normalized pattern are set to the average intensity of surrounding pixels to prevent influence of noise in the output of the filtering. The output of filtering is then phase-quantized to four levels using the Daugman method, with each filter producing two bits of data for each phasor. The output of phase quantization is chosen to be a grey code, so that when going from one quadrant to another, only 1 bit changes. The encoding process produces a bitwise template containing a number of bits of information, and a corresponding noise mask that corresponds to corrupt areas within the iris pattern, and marks bits in the template as corrupt. Since the phase information will be meaningless at regions where the amplitude is zero, these regions are also marked in the noise mask. The total number of bits in the template will be twice of the 
angular resolution times the radial resolution multiplied by the number of filters used. Here a pattern of 300-bit sequence is selected for each iris image.

\section{Support Vector Machine as a Classifier}

Support Vector Machine (SVM) is a new technique for data classification. Given a training set of instance-label pairs $\left(x_{i}, y_{i}\right), i=1,2 \ldots, l$ where $\mathrm{x}_{\mathrm{i}} \in R^{n}$ and $y \in\{1,-1\}^{l}$ the support vector machine (SVM) requires the solution of the following optimization problem [10]:

$$
\begin{aligned}
\min _{w, b, \xi} & \frac{1}{2} w^{T} w+C \sum_{i=1}^{l} \xi_{i} \\
\text { subject to } & y_{i}\left(w^{T} \phi\left(x_{i}\right)+b\right) \geq 1-\xi_{i}, \\
& \xi_{i} \geq 0 .
\end{aligned}
$$

Here, the training vectors $x_{i}$ are mapped into a higher (maybe infinite) dimensional space by the function $\phi$. Then SVM finds a linear separating hyperplane with the maximal margin in this higher dimensional space. $C>0$ is the penalty parameter of the error term. Furthermore, $K\left(x_{i}, x j\right)=\phi\left(x_{i}\right)^{T} \phi\left(x_{j}\right)$ is called the kernel function. The four basic kernel functions are given below [10]:

- Linear: $K\left(x_{i}, x_{j}\right)=x^{T}{ }_{i} x_{j}$.

- Polynomial: $K\left(x_{i}, x_{j}\right)=\left(\gamma x_{i}{ }^{T} x_{j}+r\right)^{d}, \lambda>0$.

- Radial Basis Function (RBF): $K\left(x_{i}, x_{j}\right)=\exp \left(-\gamma\left\|x_{i}-x_{j}\right\|^{2}\right), \gamma>0$.

- Sigmoid: $K\left(x_{i}, x_{j}\right)=\tanh \left(\gamma x_{i}{ }^{T} x_{j}+r\right)$.

In the present work, six iris image samples of each person from CASIA database are used to train the support vector machine. Here four kernel functions are used for experimentation and the most favorable one is selected for prediction. The remaining iris samples are used for recognition purpose.

\section{Experimental Results and Discussion}

We have used the CASIA (Chinese Academy of Sciences-Institute of Automation) iris image database and each iris class is composed of 7 samples taken in two sessions, three in the first session and four in the second. Sessions were taken with an interval of one month. Images are 320x280 pixels gray scale taken by a digital optical sensor designed by NLPR (National Laboratory of Pattern Recognition - Chinese Academy of Sciences). There are 108 classes of irises in a total of 756 iris images. Here the automatic iris and pupil detection procedure is proved as successful. For CASIA iris Database, iris region of 733 images out of 756 are detected properly 
Table 1. Accuracy of the different parts of the iris recognition system

\begin{tabular}{|c|c|c|c|}
\hline $\begin{array}{c}\text { Number of } \\
\text { sample Iris Images }\end{array}$ & $\begin{array}{c}\text { Segmentation } \\
\text { (Iris/Pupil } \\
\text { Detection) }\end{array}$ & Transformation & $\begin{array}{c}\text { Feature Extraction } \\
\& \text { Encoding }\end{array}$ \\
\hline \multirow{2}{*}{756} & 733 & 745 & 741 \\
& Accuracy & Accuracy & Accuracy $=98.01 \%$ \\
\hline
\end{tabular}

Table 2. Efficiency of various kernel functions

\begin{tabular}{|c|c|c|}
\hline Kernel Type & $\begin{array}{c}\text { No. of Support } \\
\text { Vectors }\end{array}$ & $\begin{array}{c}\text { Classification } \\
\text { Accuracy }(\%)\end{array}$ \\
\hline Linear & 678 & 93.67 \\
\hline Polynomial & 643 & 92.56 \\
\hline Radial Basis Function (RBF) & 728 & 97.34 \\
\hline Sigmoid & 347 & 91.01 \\
\hline
\end{tabular}

Table 3. Comparison of FAR and FRR for ANN and SVM

\begin{tabular}{|c|c|c|}
\hline Type of errors & FAR (\%) & FRR (\%) \\
\hline ANN & 5.8 & 1.9 \\
\hline SVM & 2.06 & .60 \\
\hline
\end{tabular}

Table 4. Average time consuming of different parts of iris recognition system

\begin{tabular}{|l|c|c|}
\hline & $\begin{array}{c}\text { Time Consuming } \\
\text { ( in ms })\end{array}$ & \% of Total Time \\
\hline Iris Segmentation & 433 & 95.16 \\
\hline Iris Image Transformation & 17 & 3.73 \\
\hline Feature Extraction & 4 & 0.87 \\
\hline Iris Classification & $<1$ & .21 \\
\hline Total & $\approx 455$ & 100 \\
\hline
\end{tabular}

which corresponds to a success rate of $96.95 \%$. The transformation procedure is also proved as successful with an accuracy rate of $98.54 \%$. However, the same pattern from images with varying amounts of pupil dilation is not perfectly reconstructed by the transformation procedure, since deformation of iris results in small changes of its surface pattern. Feature encoding is performed by 1D Gabor wavelets. Table-1 shows the accuracy of the different parts of the system. In the current research work six iris image samples of each person from CASIA are used for classification by support vector machines and the remaining iris image samples are used for recognition purpose. Four kernel functions: Linear, polynomial, RBF and sigmoid are used here and classification efficiency of each kernel is measured which is shown in Table-2 with number of support vectors found during experimentation for RBF kernel. Since the highest classification accuracy is obtained by RBF kernel, this kernel is used in our system for classification and recognition purpose. To show the effectiveness of SVM as a classifier, extracted features are also given as input to Artificial Neural 


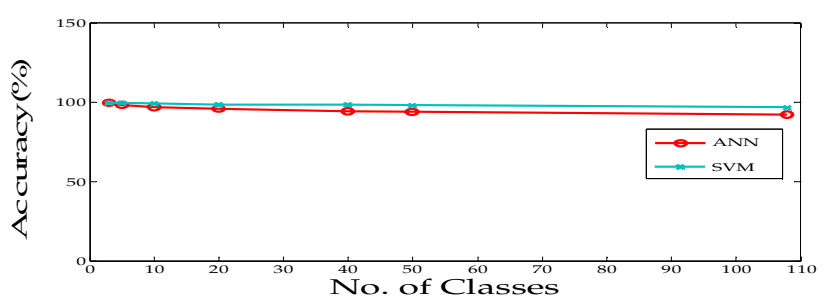

Fig. 3. Comparison of classification accuracy between ANN and SVM

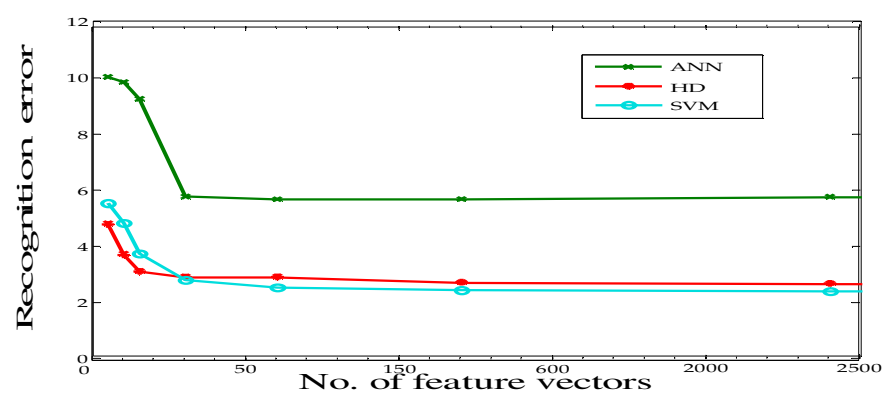

Fig. 4. Comparison between no. of feature vectors and recognition error

Network (ANN) for classification and accuracy of classification for various numbers of classes between ANN and SVM are shown in Fig.3. From this figure it is found that the performance of SVM as a classifier is far better than ANN though the classification accuracy is decreased as number of classes increased. Table-3 shows the False Accept rate (FAR) and False Reject Rate (FRR) for SVM and ANN. Finally, Table-4 indicates the time consumed by the different parts of the iris recognition system that is implemented on Pentium-4-3.00 GHz workstation. Since the total average execution time of verification process is not exceeded $455 \mathrm{~ms}$, the system is suitable for a non-intrusive authentication process. Fig. 4 shows the comparison of the feature vectors vs. recognition error among Hamming Distance (HD), ANN and SVM. In this case only RBF kernel is considered due its reasonable classification accuracy. Here SVM provides satisfactory recognition rate in comparison with HD when number of feature vectors are increased. A pattern of 300 feature vectors for each iris image is chosen for its tolerable recognition accuracy. Fig.4 also shows that SVM outperforms ANN while the classification as well as recognition accuracy is concerned.

\section{Conclusions}

In this paper an iris recognition system is implemented using support vector machine. Firstly, an automatic segmentation algorithm is applied to localize the iris region from an eye image and isolate lower, upper eyelids, and eyelashes using Hough transform. 
Next, the segmented iris region is normalized to eliminate dimensional inconsistencies between iris regions using Daugman's rubber sheet model. Finally, features of the iris are encoded by convolving the transformed iris region with 1D Log-Gabor filters and phase quantizing the output in order to produce a bit-wise biometric template. These extracted feature templates are then given to support vector machines for classification. In future, the speed of the system may be improved using the bootstrapping strategy. Also, non-symmetrical support vector machines may be used where the false accept/reject rate is treated differently.

\section{Acknowledgements}

The shared iris CASIA database is available on the web http://www.sinobiomatics. com/resources.htm. The LIBSVM (A Library for support vector machines) tool has been used in this paper for classification available at http://www.csie.ntu.edu.tw/ $\sim$ cjlin/libsvm/. This research has been supported by the NSERC, a Canada Research Chair grant and also by a grant from the Faculty of Engineering and Computer Science, Concordia University, Montreal, Canada.

\section{References}

1. J. Daugman, "How iris recognition works", Proc. of the IEEE Internat. Conf. on Image Processing, Vol. 1, pp. 33-36, 2002.

2. J. Daugman, "High Confidence Visual Recognition of Persons by a Test of Statistical Independence", IEEE Trans, on Pattern Analysis and Machine Intelligence, Vol. 15, No.11, pp.1148-1161, 1993.

3. J. Daugman, "Complete Discrete 2-D Gabor Transforms by Neural Network for Image Analysis and Compression”, IEEE Trans. Acoust., Speech, Signal Processing, Vol. 36, No. 7, pp 1169-1179, 1988.

4. R. Wildes, "Iris Recognition: An Emerging Biometric Technology", Proc. of the IEEE, Vol. 85, No. 9, pp. 1348-1363, 1997.

5. R. Bolle, J. Connell, S. Pankanti, N. Ratha, and A. Senior, "Guide to Biometrics", Springer, New York, 2003.

6. W. Kong, and D. Zhang, "Accurate Iris Segmentation Based on Novel Reflection and Eyelash Detection Model". Proc. of Internat. Sympos. On Intelligent Multimedia, Video and Speech Processing, Hong Kong, 2001.

7. W. Kong, and D. Zhang, "Detecting Eyelash and Reflections for Accurate Iris Segmentation", Internat. Journal of Pattern Recognition and Artificial Intelligence, Vol. 17, No. 6 pp 1025-1034, 2003.

8. S. Yoon, S. Choi, S. Cha, Y. Lee, and C. Tappert, "On the Individuality of Iris Biometric", ICGST Internat. Journal. On Graphics, Vision and Image Processing, Vol. 5, 2005.

9. Y. Wang, and J. Han, "Iris Recognition Using Support Vector Machines", IEEE Internet. Sympos. on Neural Network, Dalian, China, 2004.

10. N. Cristianini and J. Shawe Taylor, "An Introduction to Support Vector Machines", Cambridge University press, New York, 2000. 